\title{
Tratamento cirúrgico de cisto de colédoco: um relato de caso
}

\author{
Surgical treatment of choledocal cyst: a case report \\ Tratamiento quirúrgico del quiste de colédoco: reporte de un caso
}

Ana Clara César Coelho ${ }^{1 *}$, Andressa Silva Couto ${ }^{1}$, Daniel Assumpção Miguel ${ }^{2}$, Eduardo Rodrigues Chagas Silveira ${ }^{2}$, Fernanda Aquino de Oliveira ${ }^{1}$, José de Alencar Gonçalves de Macedo ${ }^{2}$, Matheus de Souza Nogueira ${ }^{2}$, Renata Tiemi Moreira de Resende², Ricardo Augusto Monteiro Cardoso², Sérgio Wallace Guedes Cruz².

\begin{abstract}
RESUMO
Objetivo: Descrever um caso raro de cisto de via biliar e analisar a abordagem cirúrgica complexa do cisto, evidenciando a possibilidade de um bom prognóstico e qualidade de vida a pacientes com cisto de colédoco. Relato do caso: Paciente, 14 anos, sexo feminino, sem comorbidades prévias, com quadro de icterícia, dor abdominal, hiporexia e acolia fecal. Realizados exames de imagem que identificaram massa cística localizada na cabeça do pâncreas. Encaminhada ao serviço de cirurgia geral da Santa Casa de Misericórdia de Belo Horizonte (SCMBH), onde realizou exames laboratoriais e de imagem que confirmaram o diagnóstico. Com a piora do quadro foi realizada Gastroduodenopancreatectomia e colecistectomia com reconstrução em dupla alça em Y-de-Roux. Considerações finais: A nova Classificação de Alonso Lej e Todani divide os cistos em seis diferentes tipos, variando pela topografia e apresentação do cisto. O diagnóstico precoce é de extrema importância para definir as abordagens, que se não efetuadas a tempo podem levar a graves complicações.
\end{abstract}

Palavras-chave: Cisto de colédoco, Diagnóstico, Terapêutica.

\section{ABSTRACT}

Objective: To describe a rare case of bile duct cyst and to analyze the complex surgical approach to the cyst, showing the possibility of a good prognosis and quality of life for patients with choledochal cyst. Case Report: Fourteen-year-old patient, female, with no previous comorbidities, with jaundice, abdominal pain, and exhibiting hyporexia and alcholic stools. Imaging examination identified a cystic mass located at the head of the pancreas. Patient was sent to the general surgery unit at Santa Casa de Misericórdia of Belo Horizonte (SCMBH), and imaging and laboratorial examination confirmed the diagnosis. Upon clinical worsening, patient underwent gastroduodenopancreatectomy and cholecystectomy with reconstruction in two separate loops in Roux-en-Y. Final Considerations: The most recent Todani and Alonso Lej classification divides cysts into six different types, varying by their topography and cyst presentation. It's extremely important to make an early diagnosis and take the right actions in time, in order to avoid severe complications.

Keywords: Choledocal cyst, Diagnostic, Therapeutic.

\section{RESUMEN}

Objetivo: Describir un caso raro de quiste del conducto biliar y analisar un enfoque quirúrgico complejo del quiste, mostrando la posibilidad de un buen pronóstico y calidad de vida para pacientes con quistes de colédoco. Reporte de un caso: Paciente de 14 años, sin comorbilidades previas, con cuadro clinico de ictericia, dolor abdominal, hiporexia y acolia fecal. Se le realizaron exámenes imagenologicos que identificaron una masa quística localizada en la cabeza del páncreas. Fue remitida al servicio de cirugía general de la Santa Casa de Misericordia de Belo Horizonte (SCMBH), donde se le realizaron pruebas laboratoriales y de imágen que confirmaron el diagnóstico. A medida que la afección avanzaba, el cuadro del paciente empeoró, y fue sometido a una gastroduodenopancreatectomía y colecistectomía con reconstrucción en doble alza en Roux-en-Y. Consideraciones Finales: La clasificación más reciente de Alonso Lej y Todani divide los quistes en seis tipos diferentes, que varían según su topografía y la presentación del quiste. El diagnóstico precoz es extremamente importante para definir el enfoque del tratamiento, que de no realizarse a tiempo, podria provocar complicaciones graves.

Palabras clave: Quiste de colédoco, Diagnóstico, Terapéutica.

1 Universidade de Itaúna (UIT), Itaúna - MG. *E-mail: anaclara_cesar@hotmail.com

2 Santa Casa da Misericórdia de Belo Horizonte (SCMBH), Belo Horizonte - MG. 


\section{INTRODUÇÃO}

Cistos biliares são dilatações císticas em qualquer ponto da árvore biliar, podendo ser intra e/ou extrahepáticos. Esses cistos estão associados à diversas complicações como degeneração maligna, colangite, ruptura, formação de cálculos, cirrose biliar secundária, pancreatite, entre outros (FORNY DN, et al., 2014). É uma doença rara dos ductos biliares com incidência variando de 1:100.000 a 1:150.000. Além disso, são mais comuns em mulheres, na proporção de até 4:1 em relação aos homens. Apesar das diversas tentativas, nenhuma teoria proposta sobre o mecanismo formador dos cistos biliares pode ser responsável pela formação de todos os tipos de cistos. Eles podem ser congênitos ou adquiridos e foram associados a uma variedade de anormalidades anatômicas. Alguns deles resultam da junção pancreatobiliar anormal, que permite refluxo de suco pancreático e dano ao epitélio biliar (TOPAZIAN M, 2020).

O diagnóstico é predominantemente realizado antes dos 10 anos de idade e vem aumentando devido ao aperfeiçoamento das técnicas de imagem não invasivas. A tríade clássica do cisto de colédoco (dor abdominal, icterícia e massa palpável) é mais comum em crianças que em adultos. Porém a maioria dos pacientes apresentará apenas um ou dois elementos da tríade (GONZÁLEZ GONZÁLEZ JL, et al., 2015; FORNY DN, et al., 2014).

Os cistos biliares estão associados a um risco aumentado de câncer, principalmente colangiocarcinoma. Estima-se um aumento de 20 a 30 vezes no risco de colangiocarcinoma em cistos biliares em comparação com a população em geral (TOPAZIAN M, 2020). A avaliação inicial dos cistos envolve o exame clínico juntamente com estudos laboratoriais a partir dos quais se elaboram hipóteses diagnósticas a serem posteriormente confirmadas com exames de imagem. Somente os exames laboratoriais alterados não são considerados diagnóstico, sendo que os valores anormais refletem complicações da doença (ROYCHOWDHURY N e ROY-CHOWDHURY J, 2020). Os diversos tipos de dilatações devem sofrer diferentes abordagens cirúrgicas tendo como princípio a ressecção total da dilatação e a restauração da drenagem biliar por meio da anastomose bileoentérica (PACHECO EG, et al., 2015).

Apresentamos um relato de caso de um cisto biliar do tipo I da classificação de Alonso Lej e Todani, uma enfermidade rara, de baixa incidência e prevalência. Discutiremos a apresentação clínica, a formulação diagnóstica e, com ênfase, a abordagem cirúrgica complexa diante de um cisto com distorção de anatomia gastrointestinal e de vias biliares, onde foi necessária uma conduta não convencional, pouco descrita na literatura.

O objetivo consiste em relatar um caso de abordagem cirúrgica complexa de cisto biliar e descrever a possibilidade de bom prognóstico e qualidade de vida a pacientes com cisto de colédoco.

\section{RELATO DO CASO}

Paciente de 14 anos, sexo feminino, parda, natural e procedente de Santo Antônio do Monte, MG. Procurou atendimento médico devido ao quadro de icterícia, dor abdominal difusa, hiporexia, colúria e acolia fecal há 2 meses. Nos antecedentes pessoais, nega episódios pregressos de colestase e declarou inexistência de comorbidades prévias. Realizou Ultrassonografia de Abdome Total (USG) que revelou massa cística localizada em topografia de cabeça de pâncreas causando dilatação das vias biliares intrahepáticas e hepatocolédoco com calibre aumentado. Continuou investigação com Tomografia Computadorizada (TC) identificando volumosa massa cística intra-abdominal mesogástrica, a qual determina dilatação das vias biliares e compressão das estruturas adjacentes.

Foi, então, encaminhada ao serviço de cirurgia geral da SCMBH para seguimento da propedêutica de cisto de vias biliares. $\mathrm{Na}$ admissão apresentava-se em bom estado geral, emagrecida, ictérica, com presença de massa palpável em epigástrio, com extensão para hipocôndrio direito. Em sua internação, foi realizada Ressonância Magnética de Abdome e Colangiopancreatografia por Ressonância Magnética (CPRM) que evidenciou acentuada dilatação do ducto hepatocolédoco, tendo o calibre máximo de 10,8 cm no eixo axial. Imagem compatível com cisto biliar, classificada como tipo IA de Todani. Associado a obstrução distal não significativa, condicionando dilatação das vias biliares intra e extra-hepáticas a montante (Figura 1 e 2). 


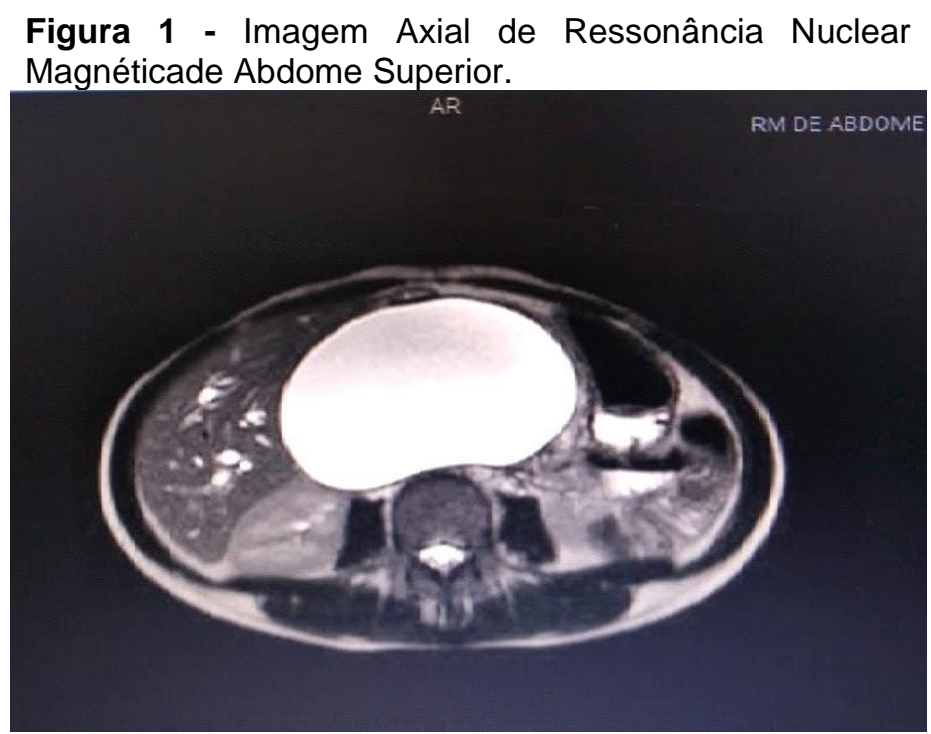

Fonte: Coelho ACC, et al., 2020.

Figura 2 - Imagem Coronal de Ressonância Nuclear Magnética de Abdome Superior.

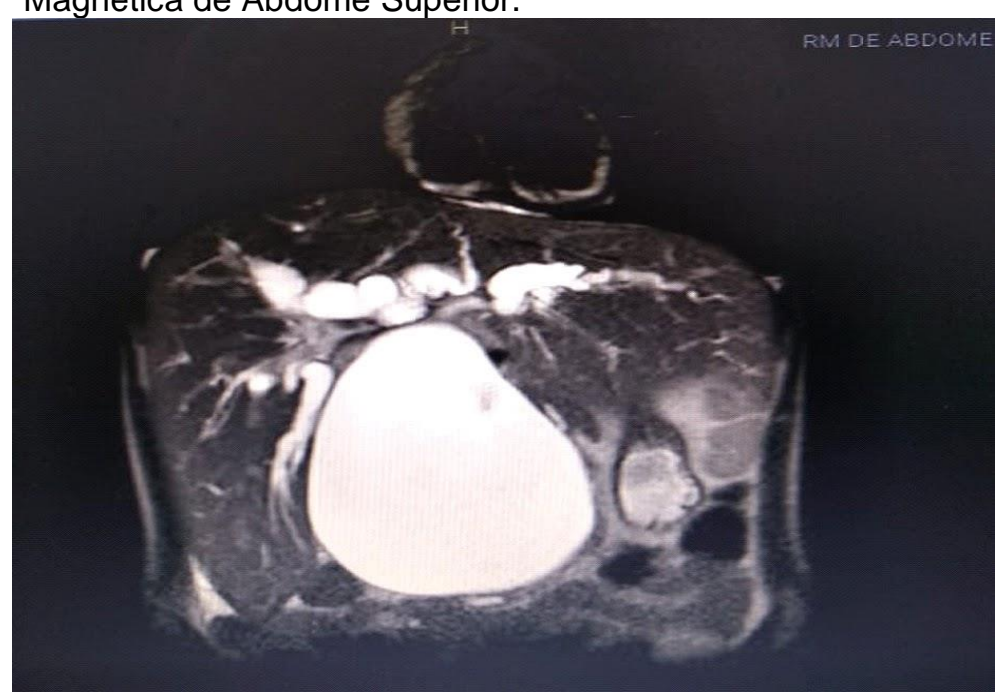

Fonte: COELHO ACC, et al. 2020.

Devido ao quadro clínico, aos achados imagiológicos e aos resultados laboratoriais [Bilirrubina Total (BT): $11,76 \mathrm{mg} / \mathrm{dL}$; Bilirrubina Direta (BD): $8,66 \mathrm{mg} / \mathrm{dL}$; Bilirrubina Indireta (Bl): $3,10 \mathrm{mg} / \mathrm{dL}$ ] a paciente foi submetida a implantação de dreno biliar percutâneo trans-hepático. Após cinco dias da implantação do dreno, paciente evoluiu com episódios de sangramento ao redor e interior do mesmo, porém sem alterações hematimétricas, mantendo bilirrubina em queda (BT: 7,40 mg/dL; BD: 3,48 mg/dL; BI:3,92 mg/dL). Avaliada por radiologia intervencionista que não indicou nova abordagem no momento, sendo mantida a proposta de derivação bileodigestiva. Recebeu, portanto, alta hospitalar para acompanhamento ambulatorial.

Passados cinco meses da sua internação, paciente compareceu a consulta no ambulatório com queixa de vômitos relacionados à obstrução do dreno biliar percutâneo. Exames laboratoriais evidenciaram aumento de enzimas hepatocíticas e canaliculares [Transaminase Glutâmico Oxalacética (TGO): 72 U/L; Transaminase Glutâmico Pirúvica (TGP): 81 U/L; Gamaglutamiltranspeptidase (GGT): 168 U/L; Fosfatase Alcalina (FA): $395 \mathrm{U} / \mathrm{L}]$. Foi internada na $\mathrm{SCMBH}$, com proposta de intervenção cirúrgica.

No dia seguinte, paciente foi submetida a ressecção através da Gastroduodenopancreatectomia associada à colecistectomia, sob anestesia geral balanceada e peridural. O procedimento foi viabilizado através da incisão subcostal bilateral (Chevron) com preservação de localização do dreno biliar percutâneo trans-hepático, seguida pela dissecção por planos. Durante o inventário da cavidade foi identificado cisto de 
via biliar volumoso, com colédoco apresentando dilatação em aproximadamente $12 \mathrm{~cm}$, ocupando todo o hipocôndrio da paciente, estendendo-se cerca de $1,5 \mathrm{~cm}$ abaixo da confluência dos hepáticos, abrangendo até a cabeça pancreática, provocando distorção anatômica significativa das estruturas do hilo hepático, estômago distal, duodeno e cabeça pancreática. Cisto compatível com achados propedêuticos, tratando-se de cisto IA na classificação de Todani (Figura 3).

Procedeu-se então, com a tentativa de dissecção de cisto coledociano em sua extensão longitudinal, sem sucesso, devido à infiltração inflamatória periductal com linfonodomegalialocorregional reacional e intensa distorção anatômica em leito de manipulação cirúrgica. Optado pela Gastroduodenopancreatectomia associada à Colecistectomia com reconstrução em dupla alça em Y-deRoux (anastomose hepaticojejunal T-L + pancreatojejunal T-L ducto-mucosa com alça transmesocólica + gastroenteroanastomose T-L pré-cólica + anastomose jejunojejunal T-L em Y-de-Roux). Por fim, implantouse dreno sentinela que foi exteriorizado através da pele. Ato realizado sem intercorrências, com envio da peça cirúrgica à análise anatomopatológica (Figura 4).

Figura 3 - Identificação e dissecção das estruturas anatômicas. Cisto IA de Todani.

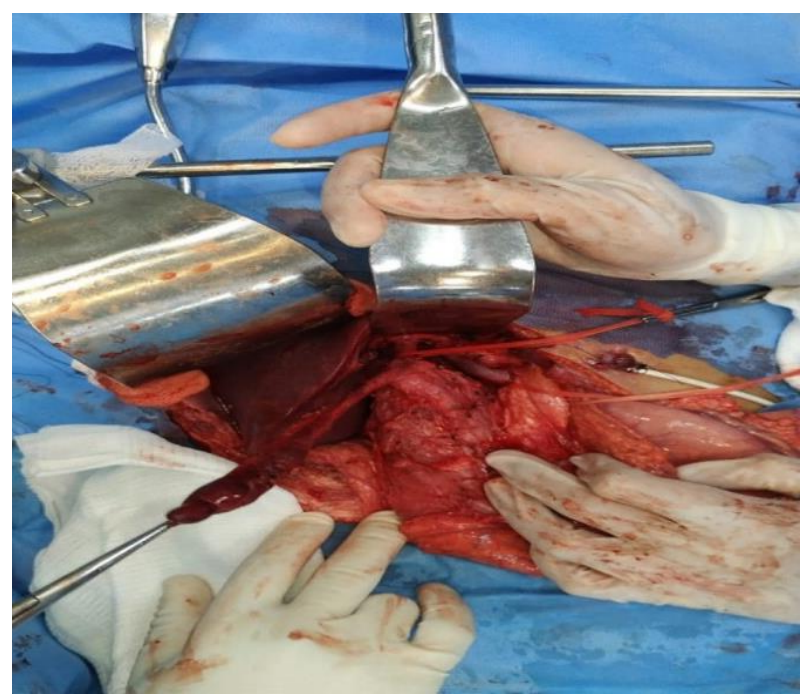

Fonte: Coelho ACC, et al. 2020.

Figura 4 - Peça cirúrgica após exérese.

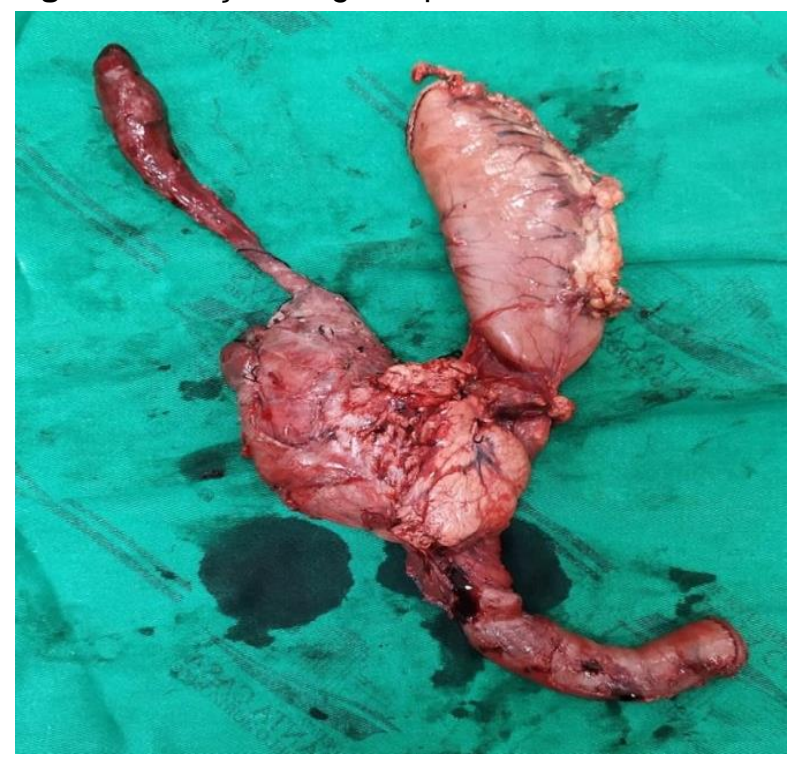

Fonte: Coelho ACC, et al. 2020. 
Após a abordagem cirúrgica, permaneceu em observação por duas semanas, período que evoluiu sem intercorrências e sem complicações pós-operatórias. Após retirada dos drenos biliar percutâneo e sentinela, recebeu alta hospitalar com melhora do estado geral. Não apresentava queixas espontâneas, tolerando dieta oral, sem relatos de náuseas e vômitos, com hábitos fisiológicos preservados, deambulando e mantendo bom padrão ventilatório.

Dois meses após sua cirurgia, assistida em ambulatório, paciente retornou à consulta, mantendo boa evolução clínica, sem intercorrências no pós-cirúrgico tardio. Relatava apetite preservado, sem novos episódios de náuseas ou vômitos. Hábitos fisiológicos preservados, sem presença de colúria e acolia fecal. Ao exame físico, a paciente apresentava-se anictérica, sem sinais de disfunção hepática, com desenvolvimento e ganho de peso adequado para a idade. Trouxe resultado de exame anatomopatológico cujo laudo evidenciou cisto de parede fibrosa revestida por epitélio sem atipias, com áreas de infiltrado inflamatório crônico, compatível com cisto de colédoco. Vesícula biliar e segmentos pancreático, gástrico e duodenal sem particularidades histológicas. Quatro linfonodos associados apresentavam hiperplasia reacional, sem sinais de especificidade ou malignidade.

\section{DISCUSSÃO}

Os cistos biliares são classificados de acordo com a localização, extensão e forma. O esquema mais aceito é a Classificação de Alonso Lej e Todani, que nos permite realizar a discussão, o diagnóstico e, principalmente, a abordagem dos cistos biliares. Os cistos do tipo I são os mais comuns (50 a $85 \%$ ) sendo limitados ao trato biliar extra-hepático. O subtipo IA, encontrado neste relato, é a dilatação cística sacular de toda via biliar extra-hepática. O subtipo IB propõe a dilatação focal de um segmento e o subtipo IC consiste na dilatação fusiforme de toda via biliar extra-hepática. Já o subtipo ID se caracteriza pela dilatação cística do ducto comum e do ducto cístico. Os cistos do tipo II (2\%) são divertículos verdadeiros dos ductos biliares extra-hepáticos. O tipo III (1 a $5 \%$ ) é a dilatação do canal biliar dentro da parede do duodeno, também chamada de coledococele. Os cistos do tipo IV (15 a 35\%) são múltiplos. O subtipo IVA envolve a via biliar intra e extra-hepática, enquanto o subtipo IVB apresenta somente acometimento extra-hepático. $\mathrm{O}$ tipo V $(20 \%)$ corresponde a cistos intra-hepáticos, também chamado de Doença de Caroli. Quando combinado com fibrose hepática recebe o nome de Síndrome de Caroli. E os cistos do tipo VI (raros) são dilatações císticas apenas do ducto cístico (TOPAZIAN M, 2020).

Sobre o caso apresentado, a principal hipótese que sustenta a formação do cisto é a junção pancreatobiliar anormal. A união anômala do ducto biliar e do ducto pancreático fora da parede duodenal, formando um longo canal comum (comprimento maior que $8 \mathrm{~mm}$ ), permite o refluxo do suco pancreático na árvore biliar ocasionando inflamação e dano ao epitélio biliar que culminaria na dilatação e formação de cisto. Embora seja uma anomalia congênita rara, está presente em 50 a $80 \%$ dos pacientes com cistos biliares (TOPAZIAN M, 2020; FORNY DN, et al., 2014).

A maioria dos cistos de colédoco apresenta seus sintomas na infância, mas por serem inespecíficos, podem não ser diagnosticados até a vida adulta. A apresentação clínica dos pacientes depende da faixa etária (GONZÁLEZ GONZÁLEZ JL, et al., 2015). Pacientes que apresentam cistos coledocianos durante a infância têm sinais e sintomas de obstrução biliar como icterícia, acolia fecal, colúria, vômitos, irritabilidade e retardo de crescimento.

Os achados de dor abdominal, icterícia e massa palpável, que compõem a tríade clássica, estavam presentes ao exame clínico da paciente em questão, corroborando com os achados da literatura, que apontam uma incidência de $85 \%$ na população pediátrica (GANDOLFI JF, et al., 2007). Os cistos das vias biliares devem ser tratados precocemente devido ao risco aumentado de câncer, principalmente colangiocarcinoma, mas também câncer de pâncreas e de vesícula biliar. A degeneração carcinomatosa tem sido associada a todos os subtipos de cistos biliares, no entanto, é mais comum nos cistos tipo I (68\%) e tipo IV (21\%) (TOPAZIAN M, 2020).

No intuito de se iniciar a investigação dos cistos, a USG é o exame de escolha pois não é invasiva e evidencia a presença de dilatações biliares. No caso de suspeita de um cisto com base na USG, a TC ou 
CPRM é normalmente o próximo passo no diagnóstico. A TC pode detectar todos os tipos de cistos biliares, examinar a relação do cisto com as estruturas circundantes e avaliar a presença de malignidade. Já a CPRM não expõe os pacientes à radiação ionizante e é o método mais sensível para a avaliação de doenças do trato biliar (73 a 100\%) (FORNY DN, et al., 2014).

O diagnóstico definitivo geralmente é realizado através da CPRM ou da Colangiopancreatografia Retrógrada Endoscópica (CPRE). A CPRE tem uma sensibilidade de até 100\% e é o padrão-ouro para o diagnóstico de junção pancreatobiliar anormal, pois fornece estudo detalhado da anatomia do trato biliar. No entanto, é um método invasivo e não isento de riscos, como a colangite e pancreatite. A vantagem da CPRM é que se trata de método não-invasivo, porém a qualidade das imagens é inferior à da CPRE (GONZÁLEZ GONZÁLEZ JL, et al., 2015).

No que tange ao tratamento, o procedimento de escolha para os cistos dos tipos I e IV é a excisão cirúrgica completa, associada à colecistectomia e reconstrução feita por hepatojejunostomia em Y-de-Roux (GANDOLFI JF, et al., 2007). No caso abordado não foi possível proceder com esta técnica devido à intensa distorção anatômica das estruturas do hilo hepático, estômago distal, duodeno e cabeça do pâncreas. A relação dos cistos tipo I com a cabeça pancreática pode complicar sua remoção e, se deixada no lugar, a porção intrapancreática de um cisto é propensa ao desenvolvimento de cálculos ou malignidade no seu remanescente (TOPAZIAN M, 2020).

Em virtude disso, optou-se pelaGastroduodenopancreatectomia e colecistectomia com reconstrução em dupla alça em Y-de-Roux. No mais, os cistos do tipo II podem ser removidos com simples excisão. Os do tipo III com excisão e esfincteroplastia. $\mathrm{E}$ os cistos do grupo $\mathrm{V}$ requerem transplante hepático. A boa evolução clínica e a baixa incidência de complicações pós-operatórias asseguram que é tecnicamente possível a realização de anastomose bileodigestiva em crianças de forma segura (FORNY DN, et al., 2014).

Embora o risco de malignidade seja diminuído em pacientes submetidos à ressecção do cisto, estes ainda apresentam risco relativo maior que o da população em geral de uma degeneração carcinomatosa. $O$ acompanhamento ambulatorial dos pacientes que foram tratados devido ao cisto biliar ainda não é bem definido. Para aqueles que foram submetidos à excisão total do cisto de forma tradicional, com hepaticojejunostomia, os testes séricos hepáticos anuais são fundamentais para rastrear estenose biliar anastomótica (TOPAZIAN M, 2020).

Os cistos de colédoco são enfermidades raras e que possuem múltiplos aspectos em sua forma. O cisto biliar tipo I de Todani, descrito neste relato de caso, é o mais comum. O diagnóstico precoce através do reconhecimento de manifestações sistêmicas, de exames laboratoriais e de imagem possibilitam a realização de um tratamento idealizado e mais apropriado. Devido a relação com o surgimento de graves complicações, como o risco de malignização tardia, o tratamento preconizado é a ressecção do cisto seguida de reconstrução do trânsito em $\mathrm{Y}$ de Roux, tendo evolução cirúrgica, clínica e metabólica satisfatórias.

No entanto, esse procedimento deve ser realizado por profissionais treinados e sob condições certas, para que sejam capazes de lidar com possíveis complicações durante o intraoperatório, principalmente nos casos em que há variações da anatomia gastrointestinal e de vias biliares, em que é necessária uma abordagem cirúrgica mais complexa e individualizada, a fim de evitar o aumento da morbimortalidade do paciente.

\section{REFERÊNCIAS}

1. ACEVEDO POLAKOVICH D, et al. Quiste de colédoco: cuadro clínico, diagnóstico y tratamiento. Acta Medica Grupo Angeles, 2015; 13(3): 171-176.

2. BOBADILLA NF, et al. Caracterización clínica de niñosconquiste de colédoco. Ene, 2016; 61(1): 11-19.

3. DE SOUZA RC, et al. Cisto de colédoco tipo I em adulto. Arquivos Médicos dos Hospitais e da Faculdade de Ciências Médicas da Santa Casa de São Paulo, 2018; 54(1): 24-27.

4. FONSECA-NETO OCLD, et al. Cisto gigante de colédoco. ABCD. Arquivos Brasileiros de Cirurgia Digestiva. São Paulo, 2007; 20(4): 297-299. 
5. FORNY DN, et al. Cisto de colédoco na infância: revisão de 30 casos. Revista do Colégio Brasileiro de Cirurgiões, 2014; 41(5): 331-335.

6. GANDOLFI JF, et al. Cisto de colédoco: relato de caso e revisão da literatura. ABCD. Arquivos Brasileiros de Cirurgia Digestiva. São Paulo, 2007; 20(2): 130-133.

7. GONZÁLEZ GONZÁLEZ JL, et al. Quiste de colédoco gigante. Revista Cubana de Cirugía, 2015; 54(2): 163-171.

8. GUERRA SC, TEIXEIRA, UF. Cisto de colédoco como achado incidental ultrassonográfico: relato de caso e revisão da literatura. ABCD. Arquivos Brasileiros de Cirurgia Digestiva, São Paulo, 2012; 25(2): 133-134.

9. GUIMARÃES FILHO A, et al. Cisto de colédoco em criança. Revista do Colégio Brasileiro de Cirurgiões, 2002; 29(1): 65-67.

10. HABER PK, SIA D. Translating Cancer Genomics for Precision Oncology in Biliary Tract Cancers. Discovery Medicine, 2019; 28(155): 255-265.

11. KHAN AS, et al. Cholangiocarcinoma: Epidemiology and risk factors. Liver International, 2019; 39:19-31.

12. LOWE R, et al. Clinical manifestations and diagnosis of cholangiocarcinoma. UpToDate, 2020.

13. HE XD, et al. The risk of carcinogenesis in congenital choledochalcystpatients: ananalysis of 214 cases. Annals of hepatology, 2014; 13(6): 819-826.

14. PACHECO EG, et al. Doenças císticas das vias biliares. Rev. Soc. Bras. Clín. Méd, 2015.

15. ROY-CHOWDHURY N, ROY-CHOWDHURY J. Diagnostic approach to the adult with jaundiceorasymptomatichyperbilirubinemia. UpToDate, 2020; 1-150.

16. SÁNCHEZ JA, et al. Quistes del colédoco. Revista Colombiana de Cirugía, 2015; 30(4): 296-305.

17. SANTAMARÍA JRG, et al. Quiste del colédoco: reporte de un caso. Rev HospJuaMex, 2005; 72(4): $161-164$.

18. TALINI C, et al. Cisto de colédoco na população pediátrica: experiência de 13 procedimentos laparoscópicos em dois anos de uma única instituição. Revista do Colégio Brasileiro de Cirurgiões, 2018; 45(3).

19. TODANI T, et al. Classification of congenital biliarycysticdisease: specialreference to typelc and IVA cysts with primaryductalstricture. Journal of Hepato-Biliary-PancreaticSurgery, 2003; 10(5): 340-344.

20. TOPAZIAN M. Biliarycysts. UpToDate. 2020. 